\title{
Epidemiological and Clinical Characteristics of 217 COVID-19 Patients in Northwest Ohio, United States
}

Mamtha Balla ${ }^{1,2}$, Ganesh Merugu ${ }^{3}$, Zeid Nesheiwat ${ }^{2}$, Mitra Patel ${ }^{4}$, Taha Sheikh ${ }^{2}$, Rawish Fatima ${ }^{2}$, Vinay K. Kotturi ${ }^{5}$, Venugopal Bommana ${ }^{1}$, Gautham Pulagam ${ }^{6}$, Brian Kaminski ${ }^{7}$

1. Internal Medicine, ProMedica Toledo Hospital, Toledo, USA 2. Internal Medicine, The University of Toledo Medical Center, Toledo, USA 3. Geriatrics, The University of Toledo Medical Center, Toledo, USA 4. Internal Medicine, The University of Toledo College of Medicine, Toledo, USA 5. Family Medicine, The University of Toledo Medical Center, Toledo, USA 6. Internal Medicine, Medical University of the Americas, Charlestown, KNA 7. Emergency Medicine, ProMedica Toledo Hospital, Toledo, USA

Corresponding author: Mamtha Balla, athamam@gmail.com

\section{Abstract \\ Background}

There is limited data on the clinical characteristics and predictors of mortality of coronavirus disease-2019 (COVID-19) in North West Ohio. We performed a retrospective review of patients hospitalized with COVID19 in the ProMedica Health System in Northwest Ohio from March 25 to June 16, 2020. The study aims to identify epidemiological, clinical characteristics, and predictors of Mortality of COVID-19 patients in Northwest Ohio.

\section{Methods}

This study was conducted on 217 COVID-19 patients admitted to ProMedica Health System Hospitals in Northwest Ohio from March 25 to June 16, 2020. We collected data, including clinical signs, symptoms, and outcomes of the COVID-19 patients. We compared clinical signs and symptoms along with comorbidities of survivors and non-survivors.

\section{Results}

Of the 217 patients included in the study, the mean age of the population was 63.13 (SD 17.8), of which 194 (89.4\%, mean age 61.7 years) survived while 23 (10.6\%, mean age 74.6 years) died. Among them, $53 \%$ were females and $47 \%$ male. Common presenting symptoms were chest pain $(91.71 \%)$, shortness of breath (79.7\%), cough (71\%), and fever (64\%). Mortality was associated with age greater than 63 (p-value 0.0052 ) and hypertension ( $\mathrm{p}$-value: 0.0058 ) with marginal significance with gender ( $\mathrm{p}$-value: 0.0642 ), chest pain ( $\mathrm{p}$ value: 0.0944 ), and history of cancer (p-value: 0.0944$)$.

Review began 03/20/2021 Review ended 04/01/2021 Published 04/05/2021

() Copyright 2021 Balla et al. This is an open access article distributed under the terms of the Creative Commons Attribution License CC-BY 4.0., which permits unrestricted use, distribution, and reproduction in any medium, provided the original author and source are credited.

\section{Conclusions}

Advanced age and hypertension (HTN) are independent predictors for increased mortality. History of cancer and chest pain are associated with increased mortality with marginal significance. Awareness among physicians about predictors of mortality is essential in dealing with COVID-19 patients. It is essential to educate the public about preventative strategies such as wearing masks to decrease mortality and morbidity from this pandemic.

Categories: Internal Medicine, Infectious Disease, Public Health

Keywords: covid-19, epidemiological findings, clinical findings, predictors of mortality, northwest ohio

\section{Introduction}

This article aims to provide a comprehensive analysis of the epidemiology and clinical characteristics of coronavirus disease-2019 (COVID-19) patients in Northwest Ohio. The novel coronavirus 2019 (2019 nCoV), also known as severe acute respiratory syndrome coronavirus two or COVID-19, originated in Wuhan, China when patients presented with atypical pneumonia unknown etiology. The initial human cases of COVID-19 were reported in December 2019. While it cannot be determined how humans in China were initially infected, evidence suggests that COVID-19 has a natural animal origin, and bats serve as its environmental reservoirs [1]. The initial cases suggested an animal to human transmission, and after an exponential increase in the number of cases, human to the human transmission had also become apparent, especially between close contacts. The primary mode of transmission is thought to be by respiratory droplets or by contact with infected secretions [2]. The World Health Organization declared this outbreak as a pandemic on March 11, 2020, the median incubation period is 5.1 days, with the onset of symptoms to occur within 11.5 days for $97.5 \%$ of the infected population [3]. The most common symptoms reported include fever, dry cough 
or chest tightness, and dyspnea; severe cases of infection can lead to pneumonia, multiple organ failure, and death [4].

There is a higher mortality rate in men than women, potentially due to sex-based immunological or gendered differences [5]. Few studies also showed higher mortality in patients with COVID-19 due to the renal involvement [6]. As of February 17, 2021, there are 824,401 confirmed cases in Ohio, with 49,788 hospitalizations, including 7,083 ICU admissions. The median age is 42 , and $46 \%$ of the infected cases are males, and $53 \%$ are females [7].

Very few studies are available in the United States mid-western region to identify predictors of Mortality, clinical signs, and symptoms of COVID-19 patients. This is one of the first studies in Midwestern, especially Northwest Ohio, to identify outcomes of COVID-19 patients based on demographics, clinical signs and symptoms. As per the studies, only with a multidirectional approach, we can mitigate COVID-19 effectively in the community as false-negative cases are prevalent [8]. As of February 24, 2021, 112,138,700 people contracted and 2,486,300 died from Coronavirus [9].

\section{Materials And Methods}

This study was conducted according to ProMedica Health System guidance and approved by the ProMedica Institutional Review Board. This is a retrospective review where we analyzed anonymous clinical data on patients with positive COVID-19 by PCR. Due to the study's characteristics, where it included only retrospective review, informed consent is waived. Data regarding epidemiological, clinical signs and symptoms were analyzed from 217 patients of COVID-19 who were admitted from March 25 to June 16, 2020. The data includes demographic (age, race, gender), epidemiological, clinical features (cough, fever, shortness of breath, loss of smell, loss of taste, gastrointestinal [GI] symptoms including nausea, vomiting, diarrhea, headache) and outcome of the patients (still in the hospital, died, or discharged). Six independent reviewers extracted data from medical records and evaluated for eligibility for the study. All the data were initially entered except the collection sheet, which has inbuilt checks to ensure data is collected appropriately. To ensure an independent evaluator assessed further $15 \%$ of all the cases (approximately 45 patients) for the date's validity and consistency. Four researchers analyzed all data. A confirmed case of COVID-19 was defined as a suspected patient with a nasopharyngeal specimen, which shows the positive result by real-time reverse transcription-polymerase chain reaction assay-RT-PCR assay. We Involved all subjects admitted to the Promedica Healthcare System and diagnosed COVID-19 infection during their admission from March to June 2020. We excluded all subjects who did not have the diagnosis of COVID-19 infection and who were not admitted to ProMedica Health System were excluded. We obtained the subjects and related information from Promedica Health system inpatient records from three hospitals including Promedica Toledo Hospital, Flower Hospital, and Bay Park Hospital. Retrospective study of the data using descriptive statistics was done. Data were summarized using descriptive statistics such as means, standard deviations, medians for numerical variables. Categorical variables were identified as frequency, percentages, cumulative frequency, and cumulative percentages. Inferential statistics such as t-tests and chi-square analyses were performed where appropriate. SPSS version 22.0 (IBM Corp., Armonk, NY) was used for all analyses. All p-values were two-tailed, and a level of $<0.05$ was considered significant.

\section{Results}

We performed this study encompassing patients living in Northwest Ohio to identify comorbid risk factors, common presenting symptoms, and overall outcomes. We compared these characteristics between survivors and non-survivors and identified the most commonly associated conditions with increasing mortality.

\section{General Information}

In 217 COVID-19 PCR positive patients who were admitted to ProMedica Health System hospitals in Northwest Ohio, the mean age of the population was 63.13 (SD 17.8), of which 194 (89.4\%, mean age 61.7 years) survived while 23 (10.6\%, mean age 74.6 years) died. Among them, 53\% were females and $47 \%$ males. Racial breakdown includes $61 \%$ white, $3 \%$ Hispanic, 35\% black, and Asian $1 \% .57 .14 \%$ of the population were obese.

\section{Clinical features of the study population}

Common presenting symptoms included chest pain (91.71\%), shortness of breath (79.7\%), cough (71\%), fever (64\%), myalgia (35\%), nausea/vomiting (32.67\%), headache (22\%,), and low appetite (21.9\%) (Table 1, Figure 1). 


\section{Cureus}

\begin{tabular}{|c|c|c|}
\hline Variables & Yes (\%) (N) & No (\%) (N) \\
\hline Anosmia & 4.29 (9) & $95.71(201)^{\star}$ \\
\hline Chest pain & $91.71(199)$ & $8.29(18)$ \\
\hline Conjunctivitis & 0 & $100(211)^{\star}$ \\
\hline Cough & $71.43(155)$ & $28.57(62)$ \\
\hline Diarrhea & $28.57(62)$ & $71.43(155)$ \\
\hline Fever & 64.06 (139) & $35.94(78)$ \\
\hline Low appetite & $21.9(46)$ & $78.1(164)^{\star}$ \\
\hline Myalgia & $35.19(76)$ & $64.81(140)^{\star}$ \\
\hline Nausea/vomiting & 32.67 (136) & $67.33(136)^{*}$ \\
\hline Ageusia & $8.61(18)$ & $91.39(191)^{\star}$ \\
\hline SOB & 79.72 (173) & $20.28(44)^{\star}$ \\
\hline Confusion & $13.82(30)$ & $86.18(187)^{\star}$ \\
\hline Headache & $22.12(48)$ & 77.88 (169) \\
\hline Rhinorrhea & $3.69(8)$ & $96.31(209)$ \\
\hline Sore throat & $5.53(12)$ & $94.47(205)$ \\
\hline ACEI & $36.41(79)$ & $63.59(138)$ \\
\hline CVD history & $41.94(91)$ & $58.06(126)$ \\
\hline History of malignancy & $8.29(18)$ & 91.71 (199) \\
\hline History of DM & $43.32(94)$ & $56.68(123)$ \\
\hline Received flu vaccine & $45(45)$ & $55(55)^{\star}$ \\
\hline History of GI issues & $24.42(53)$ & 75.58 (164) \\
\hline History of HIV & $0.92(2)$ & $99.08(215)$ \\
\hline History of HTN & 70.97 (154) & $29.03(63)$ \\
\hline NSAID usage & $31.8(69)$ & $68.2(148)$ \\
\hline History of RT disease & 39.17 (85) & $60.83(132)$ \\
\hline History of smoking & $38.25(83)$ & $61.75(134$ \\
\hline
\end{tabular}

\section{TABLE 1: Clinical characteristics and comorbid conditions of patients admitted with COVID-19.}

*Missing data is excluded from the analysis; $\mathrm{N}=$ number of the patients; $\%$ = percentage.

DM: diabetes mellitus; GI: gastrointestinal; NSAID: nonsteroidal anti-inflammatory drug; HTN: hypertension; CVD: cardiovascular disease; RT: respiratory tract; ACEI: angiotensin-converting enzyme inhibitor; SOB: shortness of breath. 


\section{Cureus}

Epidemiology and Clinical Characteristics

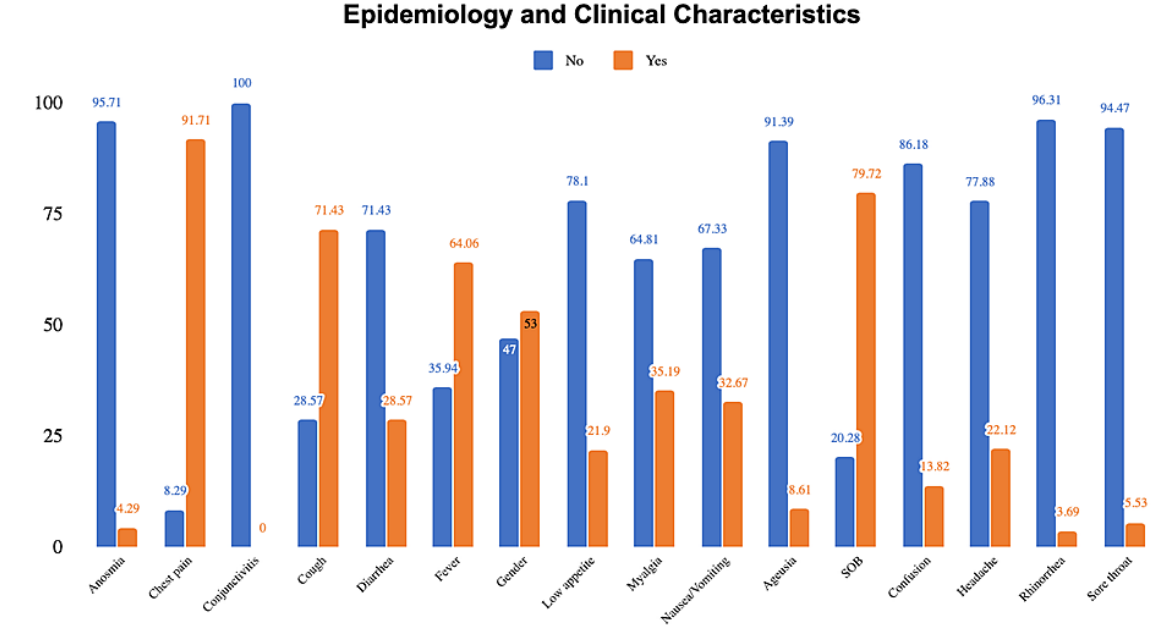

FIGURE 1: Clinical characteristics of patients admitted with COVID-19.

SOB: shortness of breath.

Comorbid conditions include diabetes mellitus (43\%) of patients, history of flu vaccine (45\%), gastrointestinal history (24.42\%), HIV (0.92\%), hypertension (HTN) (70.97\%), nonsteroidal antiinflammatory drug (NSAID) usage (31.8\%), obesity (57.14\%), angiotensin-converting enzyme inhibitor (ACEI) usage (36.4\%), history of cardiovascular disease (41.94\%), history of respiratory disease (39.17\%), smoking history (38.25\%), and cancer history (8.29\%) (Table 1, Figure 2).

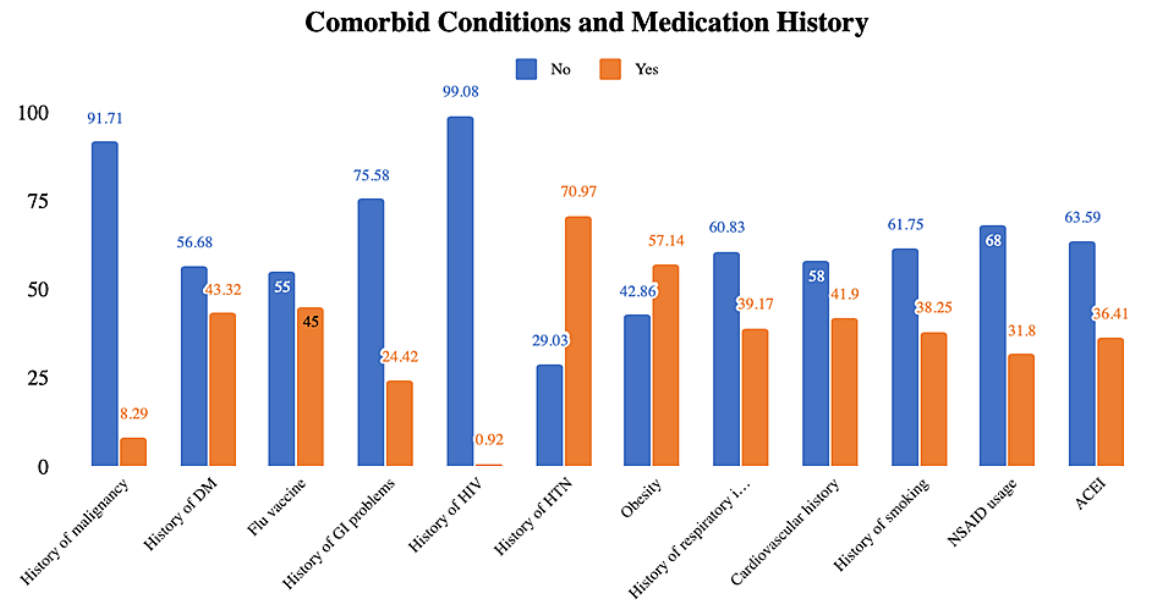

FIGURE 2: Comorbid conditions and medication history of patients admitted with COVID-19.

DM: diabetes mellitus; GI: gastrointestinal; NSAID: nonsteroidal anti-inflammatory drug; HTN: hypertension; ACEI: angiotensin-converting enzyme inhibitor.

\section{Outcome}

Of the 217 people studied, $85 \%$ were discharged, $10.6 \%$ died, $3.69 \%$ were still in the hospital, and $0.46 \%$ were transferred to higher centers. When comparing clinical characteristics and mortality, age greater 63 was statistically significant (p-value: 0.0052 ), gender and chest pain were marginally significant with p-values of 0.0642 and 0.0944 , respectively. The other variables investigated did not show any statistical significance. They were the following: anosmia (p-value: 0.2942$)$, cough (p-value: 0.8343 ), fever (p-value: 0.7363 ), low appetite (p-value: 0.9215$)$, myalgias (p-value: 0.6138 ), nausea/vomiting (p-value: 0.6840$)$, ageusia (p-value: $0.138)$, SOB (p-value: 0.7159$)$, confusion (p-value: 0.1637$)$, headache (p-value: 0.6278$)$, rhinorrhea ( $\mathrm{p}$-value: 0.3210 ), and sore throat (p-value: 0.2198 ) (Table 1 ). 
When evaluating comorbid conditions and their association with mortality, HTN was statistically significant with a p-value of 0.0058 , and there was marginal significance with cancer history (p-value: 0.0944$)$. The other variables did not show statistical significance; and included: flu vaccine (p-value: 0.2688 ), NSAID use (p-value: 0.8820), CVD history (p-value: 0.1338 ), diabetes history (p-value: 0.3647), ACE-I use (p-value: 0.5291), GI history (p-value: 0.2214), HIV (p-value: 0.6247), obesity (p-value: 0.6105 ), history of RT disease (p-value: 0.6484), and smoking history (p-value: 0.1461) (Table 2).

\begin{tabular}{|c|c|c|c|c|}
\hline Variables & & Survivors (\%) (n = 194) & Non-survivors (\%) (n = 23) & p-value \\
\hline \multirow{2}{*}{ Age } & $<63$ years & 95.33 (102) & 4.67 (5) & \multirow{2}{*}{$0.0052^{*}$} \\
\hline & $>63$ years & $83.64(92)$ & $16.36(18)$ & \\
\hline \multirow{2}{*}{ Gender } & Females & 93.04 (107) & $6.96(8)$ & \multirow{2}{*}{$0.0642^{*}$} \\
\hline & Males & $85.29(87)$ & $14.71(15)$ & \\
\hline \multirow{2}{*}{ Anosmia } & Yes & $100(9)$ & $0(0)$ & \multirow{2}{*}{0.2942} \\
\hline & No & 89.05 (179) & $10.95(22)$ & \\
\hline \multirow{2}{*}{ Cough } & Yes & 89.68 (139) & $10.32(16)$ & \multirow{2}{*}{0.8343} \\
\hline & No & $88.71(55)$ & $11.29(7)$ & \\
\hline \multirow{2}{*}{ Chest pain } & Yes & 77.78 (14) & $22.22(4)$ & \multirow{2}{*}{$0.0944^{\star \star}$} \\
\hline & No & 90.45 (180) & $9.55(19)$ & \\
\hline \multirow{2}{*}{ Fever } & Yes & $89.93(125)$ & 10.07 (14) & \multirow{2}{*}{0.7363} \\
\hline & No & $88.46(69)$ & $11.54(9)$ & \\
\hline \multirow{2}{*}{ Low appetite } & Yes & $89.13(41)$ & $10.87(5)$ & \multirow{2}{*}{0.9215} \\
\hline & No & $89.63(147)$ & $10.37(17)$ & \\
\hline \multirow{2}{*}{ Myalgia } & Yes & $90.79(69)$ & $9.21(7)$ & \multirow{2}{*}{0.6138} \\
\hline & No & $88.57(124)$ & $11.43(16)$ & \\
\hline \multirow{2}{*}{ Nausea/vomiting } & Yes & $89.39(59)$ & $10.61(7)$ & \multirow{2}{*}{0.6840} \\
\hline & No & 91.18 (124) & $8.82(12)$ & \\
\hline \multirow{2}{*}{ Ageusia } & Yes & $100(18)$ & 0 & \multirow{2}{*}{0.138} \\
\hline & No & 89.01 (170) & $10.99(21)$ & \\
\hline \multirow{2}{*}{ SOB } & Yes & 89.02 (154) & 10.98 (19) & \multirow{2}{*}{0.7159} \\
\hline & No & $90.91(40)$ & $9.09(4)$ & \\
\hline \multirow{2}{*}{ Confusion } & Yes & $96.67(29)$ & $3.33(1)$ & \multirow{2}{*}{0.1637} \\
\hline & No & 88.24 (165) & $11.76(22)$ & \\
\hline \multirow{2}{*}{ Headache } & Yes & 87.50 (42) & $12.50(96)$ & \multirow{2}{*}{0.6278} \\
\hline & No & 89.94 (152) & $10.06(17)$ & \\
\hline \multirow{2}{*}{ Rhinorrhea } & Yes & $100(8)$ & 0 & \multirow{2}{*}{0.3210} \\
\hline & No & 89 (186) & $11(23)$ & \\
\hline \multirow{2}{*}{ Sore throat } & Yes & 100 (12) & 0 & \\
\hline & No & 88.78 (182) & $11.22(23)$ & \\
\hline & Yes & 91.11 (41) & $8.89(4)$ & \\
\hline & No & $3.64(46)$ & $16.36(9)$ & \\
\hline & Yes & 85.71 (132) & $14.29(22)$ & \\
\hline & No & 98.41 (62) & 1.59 (1) & \\
\hline
\end{tabular}




\section{Cureus}

\begin{tabular}{|c|c|c|c|c|}
\hline \multirow{2}{*}{ NSAID usage } & Yes & $89.86(62)$ & $10.14(7)$ & \multirow{2}{*}{0.8820} \\
\hline & No & 89.19 (132) & $10.81(16)$ & \\
\hline \multirow{2}{*}{ CVD history } & Yes & $85.71(78)$ & $14.29(13)$ & \multirow{2}{*}{0.1338} \\
\hline & No & $92.06(116)$ & $7.94(10)$ & \\
\hline \multirow{2}{*}{ Cancer history } & Yes & 77.78 (14) & $22.22(4)$ & \multirow{2}{*}{0.0944 * } \\
\hline & No & $90.45(180)$ & $9.55(19)$ & \\
\hline \multirow{2}{*}{ Diabetes history } & Yes & $87.23(82)$ & $12.77(12)$ & \multirow{2}{*}{0.3647} \\
\hline & No & 91.06 (112) & $8.94(11)$ & \\
\hline \multirow{2}{*}{ ACEI } & Yes & 91.14 (72) & $8.86(7)$ & \multirow{2}{*}{0.5291} \\
\hline & No & 88.41 (122) & $11.59(16)$ & \\
\hline \multirow{2}{*}{ GI history } & Yes & $84.91(45)$ & 15.09 (8) & \multirow{2}{*}{0.2214} \\
\hline & No & 90.85 (149) & $9.15(15)$ & \\
\hline \multirow{2}{*}{ HIV } & Yes & $100(2)$ & 0 & \multirow{2}{*}{0.6247} \\
\hline & No & 89.30 (192) & $10.70(23)$ & \\
\hline \multirow{2}{*}{ Obesity } & Yes & 90.32 (112) & $9.68(12)$ & \multirow{2}{*}{0.6105} \\
\hline & No & $88.17(82)$ & $11.83(11)$ & \\
\hline \multirow{2}{*}{ History of RT disease } & Yes & $90.59(77)$ & $9.41(8)$ & \multirow{2}{*}{0.6484} \\
\hline & No & $88.64(117)$ & $11.36(15)$ & \\
\hline \multirow{2}{*}{ Smoking history } & Yes & $85.54(71)$ & $14.46(12)$ & \multirow{2}{*}{0.1461} \\
\hline & No & $91.79(123)$ & $8.21(11)$ & \\
\hline
\end{tabular}

TABLE 2: Clinical characteristics, comorbid conditions and their association with survival.

*Statistically significant; **marginally significant.

DM: diabetes mellitus; GI: gastrointestinal; NSAID: nonsteroidal anti-inflammatory drug; HTN: hypertension; CVD: cardiovascular disease; RT: respiratory tract; ACEI: angiotensin-converting enzyme inhibitor; SOB: shortness of breath.

\section{Discussion}

This is one of the first retrospective studies to summarize clinical features of COVID-19 patients in North West Ohio, comparing survivors and non-survivors. Our study found that patients with a history of HTN, cardiovascular disorders, and obesity were more commonly infected. When looking at symptomatology, chest pain was the most prominent symptom of COVID-19 infection with a prevalence of $91.71 \%$ but was also marginally associated with death (p-value: 0.0944). Besides, we found that most patients who did not survive were greater than 63 years old (p-value: 0.0052). Also, patients with HTN were at an increased risk of both infection and death (p-value: 0.0058). Similar studies have also shown this correlation between HTN and susceptibility to COVID-19 [10-12]. Surprisingly, males and those with a cancer history were associated with increased odds of death with marginally significant p-values of 0.0642 and 0.0944 , respectively.

When compared to other studies, we found a higher rate of chest pain in our patient population. Previous studies from China and Korea report a low rate of chest pain symptomatology in their patient population [13-15]. This is surprising and may be due to a late presentation of COVID-19 and subsequent late complications such as pericarditis and myocarditis [16]. There was an effort to discourage patients from coming to the hospital unless necessary to reduce their infection risk. It could be possible that many of those patients did not seek treatment until it was late into the course. Our data also showed CVD, HTN, and obesity associated with COVID-19 infection, similar to other U.S. studies $[17,18]$.

In contrast to recent studies, however, our patient sample has a low prevalence of anosmia at $4.29 \%$. In a recent study from Italy, $64 \%$ of symptomatic COVID-19 patients had anosmia, and again in a similar study from Iran, $98.3 \%$ of their infected patients had anosmia $[19,20]$. The mechanism for anosmia related to COVID-19 is not yet known. Still, a theory suggests cell disruption of the nasal epithelium, causing a 
proinflammatory state of the olfactory neuroepithelium that causes cell disruption to be the cause of anosmia [21]. Currently, we do not have any robust theories on why our patient population differs in this aspect of symptomatology.

Another interesting symptom that was not as apparent in our study compared to others was the fever history. With $64.06 \%$ of our patient sample showing the clinical characteristic of fever, this is relatively low compared to other studies. Previous studies note over $85 \%$ of their patient sample with fever $[17,22,23]$. However, fever's presence did not appear to have any statistical significance compared to mortality risk (pvalue: 0.7363 ). Lastly, G.I. symptoms such as nausea/vomiting (32.67\%) and diarrhea (28.57\%) were similarly low compared to previous studies in New York, the epicenter of COVID-19 in the United States and China [24]. We also did not notice a lot of central or peripheral neurological symptoms [25-27]. This suggests that coronavirus is primarily a respiratory-driven virus without much involvement of the GI tract.

Accurately identifying the epidemiology and clinical characteristics of COVID-19 infection can help clinicians understand the disease course, susceptibility, and prognosis of patients with COVID-19 infection. Our findings in this study mostly correspond to prior studies indicating increased susceptibility for older patients, HTN, DM, and obesity. One interesting thing which we noted that, increased prevalence of chest pain with COVID-19 infection. And statistically, it is associated with increased mortality (with marginal significance).

\section{Limitations}

It is a retrospective study. The small sample size may not accurately depict the general population. Also, we cannot determine causation but only association. Lastly, it is difficult to rule out any confounders given the methodology of this study, and temporal relationships are challenging to assess. Larger studies are needed to identify mortality predictors, especially concerning the history of chest pain and cancer history.

\section{Conclusions}

COVID-19 is a massive pandemic of the century, with more than 20 million infected and over 700,000 deaths. This retrospective cohort study investigates the epidemiology and characteristics of 217 patients of COVID-19 in Northwest Ohio, United States. Our findings show that age >63, male sex, HTN history, cancer history, and chest pain on admission may be significant predictors of COVID-19 outcomes.

\section{Additional Information}

\section{Disclosures}

Human subjects: Consent was obtained or waived by all participants in this study. ProMedica Institutional Review Board issued approval IRB \#20-055:. April 18, 2020 Mamtha Balla, MD RE: IRB \#20-055: Outcomes in COVID-19 patients based on demographics, clinical signs \& symptoms, hematological and radiological parameters - In Northwest Ohio-A Retrospective study Dear Dr. Balla: I have reviewed and approved your application for expedited review of the new study listed above. Your study is eligible for expedited review Category \#5) Research involving materials (data, documents, records, or specimens) that have been collected, or will be collected solely for non-research purposes (such as medical treatment or diagnosis). (NOTE: Some research in this category may be exempt from the HHS regulations for the protection of human subjects. 45 CFR 46.101(b)(4). This listing refers only to research that is not exempt.). Approval includes the following documents: $\mathbf{X}$ IRB Protocol Proposal - Balla.docx (Protocol) $\mathbf{X}$ Retrospective data collection layout.xlsx (Data Collection Form) $\mathbf{\nabla}$ Waiver of Authorization 6-14-19-IRB.docx (Waiver of Authorization) If applicable, the following apply to your study: $\mathbf{\nabla}$ Full waiver You are granted permission to conduct your study as described in your application effective immediately. This study will expire on: April 17, 2021 Although the ProMedica IRB has granted you permission to conduct your study as described in your application, you may be subject to further appropriate review and approval or disapproval by officials of the institution ( 45 CFR 46.112 \& 50 CFR 56.112). Please note that any changes to the study must be promptly reported and approved by the PHS IRB before being implemented. Some changes may be approved by expedited review ( 45 CFR 46.110 \& 50 CFR 56.110); others require full board review. If you have any questions or require further information, please contact the ProMedica IRB Office, at (419) 291-5362 or email: phsirb@promedica.org. Sincerely, Electronically signed by Lynnea Lau on 4/18/2020 6:49:49 PM Director and Member, ProMedica Institutional Review Board. Animal subjects: All authors have confirmed that this study did not involve animal subjects or tissue. Conflicts of interest: In compliance with the ICMJE uniform disclosure form, all authors declare the following: Payment/services info: All authors have declared that no financial support was received from any organization for the submitted work. Financial relationships: All authors have declared that they have no financial relationships at present or within the previous three years with any organizations that might have an interest in the submitted work. Other relationships: All authors have declared that there are no other relationships or activities that could appear to have influenced the submitted work.

\section{Acknowledgements}

We thank Dr. Vijay Gayam for the final critics and Divya Balla for the article's final proofreading. Appreciate 
Dr. Kaminski for their valuable time in doing this project. We thank IRB and IT departments in Promedica Toledo Hospital for their support during this project. Appreciate all the authors who contributed to collecting the data, analyzing, and writing this manuscript

\section{References}

1. Lake MA: What we know so far: COVID-19 current clinical knowledge and research . Clin Med. 2020, 20:124127. 10.7861/clinmed.2019-coron

2. Guo YR, Cao QD, Hong ZS, et al.: The origin, transmission and clinical therapies on coronavirus disease 2019 (COVID-19) outbreak - an update on the status. Mil Med Res. 2020, 7:11. 10.1186/s40779-020-00240-0

3. Lauer SA, Grantz KH, Bi Q, et al.: The incubation period of coronavirus disease 2019 (COVID-19) from publicly reported confirmed cases: estimation and application. Ann Intern Med. 2020, 172:577-582. 10.7326/M20-0504

4. Park M, Cook AR, Lim JT, Sun Y, Dickens BL: A systematic review of COVID-19 epidemiology based on current evidence. J Clin Med. 2020, 9:10.3390/jcm9040967

5. Wenham C, Smith J, Morgan R: COVID-19: the gendered impacts of the outbreak. Lancet. 2020, 395:846848. 10.1016/S0140-6736(20)30526-2

6. Adapa S, Chenna A, Balla M, et al.: COVID-19 pandemic causing acute kidney injury and impact on patients with chronic kidney disease and renal transplantation. J Clin Med Res. 2020, 12:352-361. 10.14740/jocmr4200

7. Ohio Department of Health Coronavirus (COVID-19) . (2021). Accessed: February 17: https://coronavirus.ohio.gov/wps/portal/gov/covid-19/home.

8. Balla M, Merugu GP, Pokal M, Gayam V, Adapa S, Naramala S, Konala VM: A comprehensive approach is vital for diagnosing COVID-19: a case of false negative. J Clin Med Res. 2020, 12:315-319. 10.14740/jocmr4173

9. Coronavirus Map: Tracking the Global Outbreak . (2021). Accessed: February 24: https://www.nytimes.com/interactive/2020/world/coronavirus-maps.html.

10. Lian J, Jin X, Hao S, et al.: Epidemiological, clinical, and virological characteristics of 465 hospitalized cases of coronavirus disease 2019 (COVID-19) from Zhejiang province in China. Influenza Other Respir Viruses. 2020, 14:564-574. 10.1111/irv.12758

11. Nie Y, Li J, Huang X, et al.: Epidemiological and clinical characteristics of 671 COVID-19 patients in Henan Province, China. Int J Epidemiol. 2020, 49:1085-1095. 10.1093/ije/dyaa081

12. Argenziano MG, Bruce SL, Slater CL, et al.: Characterization and clinical course of 1000 patients with coronavirus disease 2019 in New York: retrospective case series. BMJ. 2020, 369:m1996. 10.1136/bmj.m1996

13. Kim GU, Kim MJ, Ra SH, Lee J, Bae S, Jung J, Kim SH: Clinical characteristics of asymptomatic and symptomatic patients with mild COVID-19. Clin Microbiol Infect. 2020, 26:948.e1-948.e3. 10.1016/j.cmi.2020.04.040

14. Chen N, Zhou M, Dong X, et al.: Epidemiological and clinical characteristics of 99 cases of 2019 novel coronavirus pneumonia in Wuhan, China: a descriptive study. Lancet. 2020, 395:507-513. 10.1016/S01406736(20)30211-7

15. Yan Y, Yang Y, Wang F, et al.: Clinical characteristics and outcomes of patients with severe covid-19 with diabetes. BMJ Open Diabetes Res Care. 2020, 8:10.1136/bmjdrc-2020-001343

16. Inciardi RM, Lupi L, Zaccone G, et al.: Cardiac involvement in a patient with coronavirus disease 2019 (COVID-19). JAMA Cardiol. 2020, 5:819-824. 10.1001/jamacardio.2020.1096

17. Aggarwal S, Garcia-Telles N, Aggarwal G, Lavie C, Lippi G, Henry BM: Clinical features, laboratory characteristics, and outcomes of patients hospitalized with coronavirus disease 2019 (COVID-19): Early report from the United States. Diagnosis. 2020, 7:91-96. 10.1515/dx-2020-0046

18. Suleyman G, Fadel RA, Malette KM, et al.: Clinical characteristics and morbidity associated with coronavirus disease 2019 in a series of patients in metropolitan Detroit. JAMA Netw Open. 2020, 3:e2012270. 10.1001/jamanetworkopen.2020.12270

19. Spinato G, Fabbris C, Polesel J, Cazzador D, Borsetto D, Hopkins C, Boscolo-Rizzo P: Alterations in smell or taste in mildly symptomatic outpatients with SARS-CoV-2 infection. JAMA. 2020, 323:2089-2090. 10.1001/jama.2020.6771

20. Moein ST, Hashemian SM, Mansourafshar B, Khorram-Tousi A, Tabarsi P, Doty RL: Smell dysfunction: a biomarker for COVID-19. Int Forum Allergy Rhinol. 2020, 10:944-950. 10.1002/alr.22587

21. Whitcroft KL, Hummel T: Olfactory dysfunction in COVID-19: diagnosis and management. JAMA. 2020, 323:2512-2514. 10.1001/JAMA.2020.8391

22. Zhong ZF, Huang J, Yang X, et al.: Epidemiological and clinical characteristics of COVID-19 patients in Hengyang, Hunan Province, China. World J Clin Cases. 2020, 8:2554-2565. 10.12998/wjcc.v8.i12.2554

23. Balla M, Merugu GP, Patel M, et al.: COVID-19, modern pandemic: a systematic review from front-line health care providers' perspective. J Clin Med Res. 2020, 12:215-229. 10.14740/jocmr4142

24. Liang WH, Guan WJ, Li CC, et al.: Clinical characteristics and outcomes of hospitalised patients with COVID-19 treated in Hubei (epicentre) and outside Hubei (non-epicentre): a nationwide analysis of China. Eur Respir J. 2020, 55:10.1183/13993003.00562-2020

25. Malayala SV, Raza A: A case of COVID-19-induced vestibular neuritis . Cureus. 2020, 12:e8918. 10.7759/cureus.8918

26. Vanaparthy R, Malayala SV, Balla M: COVID-19-induced vestibular neuritis, hemi-facial spasms and Raynaud's phenomenon: a case report. Cureus. 2020, 12:e11752. 10.7759/cureus.11752

27. Atluri P, Vasireddy D, Malayala S: COVID-19 encephalopathy in adults. Cureus. 2021, 13:e13052. 10.7759/cureus.13052 